\title{
Integrated Simultaneous Chemical, Surface Potential, and Topographic Imaging at $<10$ nm Spatial Resolution with Peak Force Infrared - Kelvin Probe Force Microscopy
}

Xiaoji $\mathrm{Xu}$ and Devon Jakob

Lehigh University, Bethlehem, Pennsylvania, United States

Multimodal measurements of chemical composition, electrical properties, mechanical properties, and topography by scanning probe microscopy (SPM) deliver correlations across properties at the nanoscale, and provide clues to the structure-function relationship of materials. In the past, measurements with these modalities are operated separately with different operational modes of SPM. Not only the sequential measurements require additional operation time, are subject to scanner/sample drift, but also different modalities of SPM have different spatial resolutions, which undermine correlative analysis. For example, the popular frequency-modulated Kelvin Probe Force Microscopy measures the surface potential with 30 50 nm spatial resolution under the ambient conditions, whereas the SPM measurements of chemical composition, mechanical properties, and topography can routinely achieve $<10 \mathrm{~nm}$ spatial resolution. Correlative analysis of quantities at $30 \mathrm{~nm}$ spatial resolution with quantities of $10 \mathrm{~nm}$ resolution would not provide the full insight.

At the conference, we will present our invention of an integrated SPM mode that can simultaneously provide chemical, surface potential, mechanical, and topographic imaging at $<10 \mathrm{~nm}$ spatial resolution under the ambient conditions. We name it peak force infrared-Kelvin probe force microscopy (PFIRKPFM), as we achieved it through an integration of peak force infrared microscopy and pulsed force Kelvin probe force microscopy. The peak force infrared microscopy is an action-based photothermal infrared microscopy that operates in the peak force tapping mode. The pulsed force Kelvin probe force microscopy is a recently invented peak force tapping based technique that maps the surface potentials of the sample surface at sub $10 \mathrm{~nm}$ spatial resolution. Our work seamlessly integrates these two peak force tapping based imaging techniques. In a single scan, the integrated PFIR-KPFM delivers simultaneous multimodal measurement at a comparable and high spatial resolution of $<10 \mathrm{~nm}$. As a demonstration, we measured a naturally-degraded $\mathrm{CH}_{3} \mathrm{NH}_{3} \mathrm{PbBr}_{3}$ perovskite single crystal and a laser-induced degraded crystal. AFM topography, mechanical modulus, contact potential difference (CPD), and nano-IR imaging at infrared absorption of perovskite are simultaneously acquired and are displayed in Figure 1. The multimodal measurements reveal different correlation patterns for the two degradation pathways. The PFIR-KPFM is also applicable to other nanoscale heterogeneous materials, from protein structures, ferroelectric materials, to organic photovoltaics and semiconductors. 


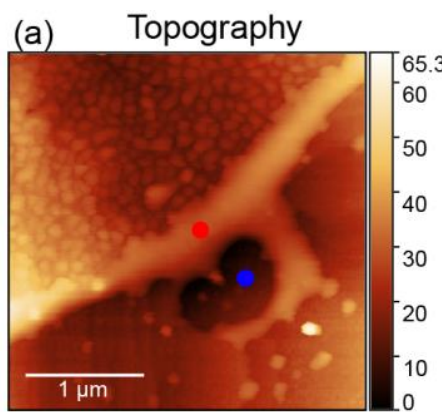

(c) Contact potential difference

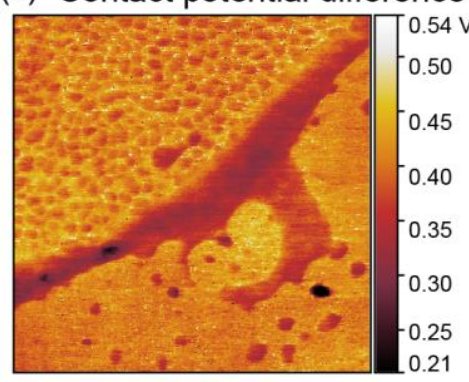

(b) Mechanical modulus

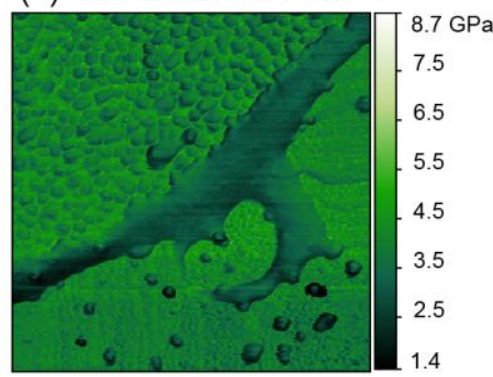

(d) Nano-IR imaging @1585 cm-1

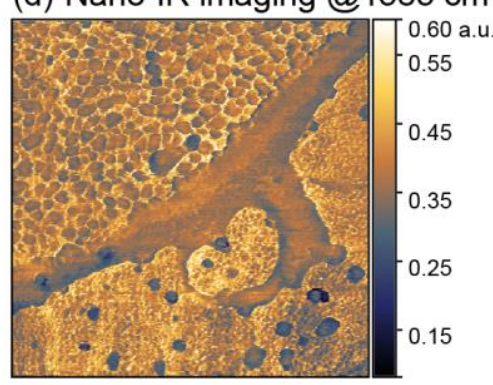

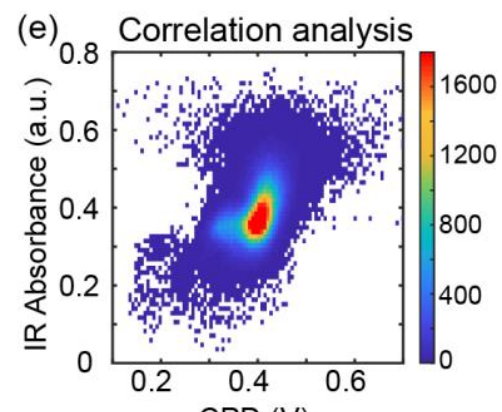

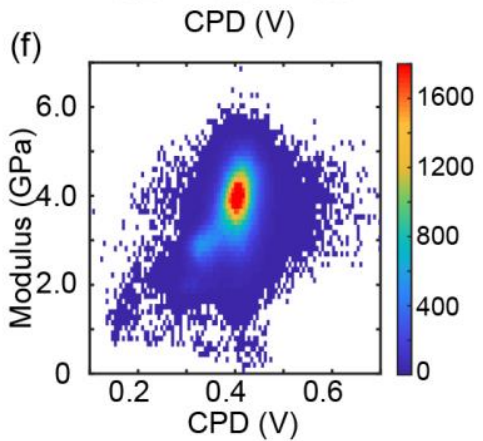

Figure 1. PFIR-KPFM measurement of a naturally degrade $\mathrm{CH} 3 \mathrm{NH} 3 \mathrm{PbBr} 3$ perovskite single crystal. (a) topography. (b) Modulus. (c) CPD. (d) The nano-IR image at the infrared frequency of $1585 \mathrm{~cm}-1$ (e) Correlation between CPD and IR signal. (f) Correlation between CPD and modulus.

\section{References}

Le Wang, Haomin Wang, Martin Wagner, Yong Yan, Devon S. Jakob and Xiaoji G. Xu "Nanoscale Simultaneous Chemical and Mechanical Imaging via Peak Force Infrared Microscopy" Science Advances 3, e1700255 (2017)

Le Wang, Martin Wagner, Haomin Wang, Siuling Pau-Sanchez, Jiahan Li, James H. Edgar, and Xiaoji G. $\mathrm{Xu}$ "Revealing Phonon Polaritons in Hexagonal Boron Nitride by Multi-Pulse Peak Force Infrared Microscopy" Advanced Optical Materials, 1901084 (2019)

Devon S. Jakob, Le Wang, Haomin Wang, and Xiaoji G. Xu "Spectro-mechanical Characterization of Aromaticity and Maturity of Kerogens in Oil Shale at $6 \mathrm{~nm}$ Spatial Resolution" Analytical Chemistry, 91, 14, 8883 (2019) 